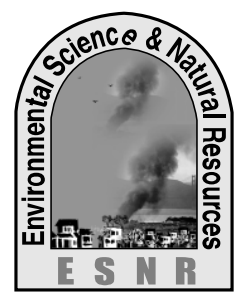

\title{
Morphological Characterization of Two Fresh Water Eels Monopterus cuchia (Hamilton, 1822) and Ophisternon bengalense (McClelland, 1844)
}

\author{
P. R. Roy, N. S. Lucky* and M. A. R. Hossain \\ Department of Fisheries Biology and Genetics, \\ Bangladesh Agricultural University, Mymensingh \\ *Corresponding author: nahidlucky@gmail.com
}

\begin{abstract}
Morphometric and meristic characters and truss measurements of 32 Monopterus cuchia, and of 17 Ophisternon bengalense were compared to know the population status of two fresh water eels of Bangladesh. The mean numbers of line below head were significantly different between two species. Significant differences were observed in 11 morphometric characters: Pre dorsal length (PDL), Post dorsal length (PoDL), Post anal length (PoAL), Head length (HL), Snout length (SnL), Upper jaw length (UJL), Lower jaw length (LJL), Head width (HW), Pre orbital length (PrOrL), Least body diameter (LBD) and Highest body diameter (HBD) and one truss measurement (3-5) between two species in varying degrees. For both morphometric and landmark measurements, the first and second DF (discriminant function) accounted $64.8 \%$ and $33.2 \%$ of among group variability, explaining $98 \%$ of total group variability. M. cuchia collected from Mymensingh and from Dinajpur constructed one sub-cluster and $O$. bengalense collected from Sathkhira and from Bagerhat constructed another sub-cluster based on the Distance of squared Euclidean dissimilarity. A correct classification of individuals into their original population from leave-one-out-classification varied between $93.3 \%$ and $94.1 \%$ by discriminant analysis. The results of the present study suggested that there was limited intermingling among populations and the populations of the species were separated from one another.
\end{abstract}

Key words: Meristic characters, Morphometric characters, Population structure, Truss measurements

\section{Introduction}

The mud eel, Monopterus cuchia (Hamilton) is freshwater air breathing fish, locally known as Cuchia, belongs to the family Synbranchidae of the Order Synbranchiformes. It is commonly found in the freshwater of Bangladesh, Pakistan, Northern and Northeastern India and Nepal (Jingran and Talwar, 1991). They have high fecundity, and are protogynous hermaphrodite. Once, indigenous mud eel, M. cuchia was abundant throughout the Bangladesh. They were available in plenty in mud holes in shallow "beels" and "boro" paddy field particularly in Sylhet, Mymensingh and Tangail Districts (Rahman, 2005). Now-a-days this fish is hardly found in the open water system due to over exploitation and various ecological changes in its natural habitat. The swamp eel also known as Bengal eel, $O$. bengalense is a freshwater, demersal and brackish water eel, belongs to the family Synbranchidae of the Order Synbranchiformes. It is distributed in India, Sri Lanka, Indonesia, Philippines and New Guinea. Adults inhabit both fresh and brackish waters of rivers and swamps or near the river mouth. The male guards and builds nest or burrow (Breder and Rosen, 1966). Because of its taste $O$. bengalense is a fish of high demand to the tribal people of Bangladesh and people of China, Hong Kong, Thailand, Vietnam, Malaysia, Japan and Indonesia.

Globally eel production was expected to grow by thrice between 1985 and 1992 which representing an increase of about 58\% (ADCP, 1995). World aquaculture production of freshwater eels has increased over the past decade and is currently around 2, 33,000 $\mathrm{MT}^{-1}$ year which valued at over US\$975 million (FAO, 2005; FAO/UN, 2005). A significant commercial eel fishery exists in various countries like Australia, Thailand, Malaysia, Japan,
Korea, USA, China, Italy, Greece, Egypt, Singapore, Cambodia and Taiwan (ADCP, 1995; Hicks and Mc Caughan, 1997; August and Hicks, 2006) consisting a great available export market (Ishak, 1994; Moriarty and Dekker, 1997; Jessop, 2000). Shrimp, Bangladesh's main aquatic export item facing grave environmental, sociopolitical and socio-economic consequences have resulted in the wake of its expansion which jeopardized the livelihoods of millions, particularly the most vulnerable women and children (Mazid, 2002). Concurrently, crab is gaining an important position of immense prospects. However, eels, a recent export item, though, have not yet been give any attention of its culture and collection could be considered as an alternative option for poor peoples. Collection from the wild to meet growing export demand and lacking of aquaculture of this species could be the major concern for biodiversity loss in Bangladesh. However, considering the increasing demand in the international markets (Usui, 1991; VAC, 1999; FAO, 2005), eel fishery has been gaining popularity among the coastal community of greater Khulna and Chittagong regions as well as greater Mymenshing and Shylet. Five species of eel, M. cuchia, Anguilla bengalensis, $O$. bengalense, Pisodonophis boro and Pisodonophis cancrivorus (Chowdhury et al., 1980; FRSS, 1984; BOBP, 1985) are available in Bangladesh, in which, $M$. cuchia, $P$. boro and $O$. bengalense are presently exported to Japan, Korea, Hong Kong, Thailand, China and Taiwan from Bangladesh. Mud eel exported in a large quantity to China and other Asian countries, collecting from wild, and no fry production and culture is practiced yet. On the other hand, plenty of works on reproductive physiology, neuroendocrine mechanism of reproduction, fry production and aquaculture of its close relative from the 
same genus (swamp eel, $M$. albus) have already been done in many countries and eels have been practiced intensive aquaculture for its high market value. Since there is very little culture system for freshwater eels, it is necessary to develop a scientific eel culture system. Advanced aquaculture is not possible without proper understanding of the various biological factors of fishes such as morphological study, food and feeding habit, hematology, reproductive biology, growth and water quality parameters which are responsible directly for the production of biomass in a water body.

The eel aquaculture industry in Bangladesh is completely absent, only capture based fishery practice are performed. Both freshwater and saltwater eels of Bangladesh could be grown for international market. Hence, Bangladesh has great opportunity to develop eel farming industry and to enter those European and Asian markets, if proper attempt could be taken. Although some laboratory-scale progress has been made in maturing and fertilizing the eggs of some species of eels, it has not yet been studied with the whole morphological characterization of eel.

To better understand and document morphological variation in eel especially $M$. cuchia and $O$. bengalense, their head and body shape and color pattern, their whole physical characteristics and comparison between them is essential. Populations in close geographic proximity may represent separate introductions of genetically distinct forms thus has significant management implications. Thus the present study was conducted to characterize and compare the two species of eels, M. cuchia and $O$. bengalense.

\section{Materials and Methods}

\section{Collection of sample}

Monopterus cuchia were collected from two places of Bangladesh, Dinajpur and Mymensingh and Ophisternon bengalense were collected from two places, Satkhira and Bagerhat.

\section{Rearing of sample}

Total $32 \mathrm{M}$. cuchia and $17 \mathrm{O}$. bengalense were reared in two tanks at the mini hatchery under the Faculty of Fisheries, Bangladesh Agricultural University, Mymensingh. The fishes were reared maintaining appropriate water quality, fed with live earthworm and tubifex twice a day. Small parts of bamboos were placed in the tanks for their shelter.

\section{Measurement of morphometric and meristic characters}

Twenty six morphometric characters: Total length (TL), Pre dorsal length (PDL), Post dorsal length (PoDL), Pre anal length (PAL), Post anal length (PoAL), Length of lateral line (LAL), Head length (HL), Snout length(SnL), Upper jaw length (UJL), Lower jaw length (LJL), Mouth gape (MG), Eye diameter (ED), Head depth (HD), Head width (HW), Pre orbital length(POL), Post orbital length
(PoOrL), Greatest body depth (GBD), Least body depth (LBD), Greatest width of body (GWB), Highest body diameter (HBD), Width of body at vent (WBV), Depth of body at vent (DBV), Distance between vent and commencement of dorsal fin (DBCB), Intestine length (IL), Fat length (FL), Length beside lateral line (LBLL) of the fish were measured using vernier calipers with an accuracy of $0.05 \mathrm{~mm}$. A total of five meristic characters: No. of body line, No. of line below head, No. of teeth on upper jaw, No. of teeth on lower jaw and No. of gill raker were also analyzed.

\section{Land mark distances of the species}

The truss network system described for fish body morphometrics (Hossain et al., 2010) was used to construct a network on fish body, 6 landmarks determining 8 distances were produced and measured as illustrated in Fig. 1. Each landmark was obtained by placing the fish on a graph paper and then the landmark points were detected with colored pointers. Finally the distances on the graph paper were measured using vernier calipers.

After measurement of morphometric, meristic and landmark distances, the fish were dissected using scissor and tweezers. Intestine of the fish were cleaned with fresh tap water and were measured and were also try to seen the sex pattern. At the head position head was dissected and takeout the gill raker and both upper jaw and lower jaw are cut and count the teeth.

\section{Statistical analyses}

Sexes were determined by microscopic examination of the respective gonads and this subset was used to test hypothesis of no sexual dimorphosim in morphometric, landmark distance and meristic characters of both the species. Sexual variation was analyzed using independent sample $t$-test.

A multivariate discriminant analysis was used for morphometric data to identify the combination of variables that best separate both the species. Prior to the analysis, size effects from the data set were eliminated. Variations were attributed to body shape differences, and not to the relative size of the fish. In the present study, there were significant linear correlations among all measured characters and the total length of the fish. Therefore, it was necessary to remove size-dependent variation for all the characters. An allometric formula given by Elliott et al. (1995) with slight modification was used to remove the size effect from the data set.

$\mathrm{M}_{\mathrm{adj}}=\mathrm{M}\left(\mathrm{L}_{\mathrm{s}} / \mathrm{L}_{\mathrm{o}}\right)^{\mathrm{b}}$

where,

$\mathrm{M}=$ Original measurement, $\mathbf{M}_{\mathrm{adj}}=$ Size adjusted measurement, $\mathrm{L}_{\mathrm{o}}=$ Total length of fish and $\mathrm{L}_{\mathrm{s}}=$ overall mean of total length 
Parameter $b$ was estimated for each character from the observed data as the slope of the regression of $\log \mathrm{M}$ on $\log \mathrm{L}_{\mathrm{o}}$, using all fish in all groups. The efficiency of size adjustment transformations was assessed by testing the significance of the correlation between transformed variable and total length.

Efficiency of the allometric formula in removing size effect from the data was justified by using correlation between total length and the adjusted morphological and meristic characters and landmark distances. Total length were excluded first and not transformed because using this parameter as standard all other parameters were standardized. After the allometric transformation, the correlation results revealed that all of the meristic variables studied were free from the influence of size. The degree of similarity among samples in the overall analysis and relative importance of each measurement for group separation were assessed by discriminant function analysis (DFA) with cross validation. Population centroids with $95 \%$ confidence ellipses derived from the DFA were used to visualize relationships among the individuals of groups. A dendrogram of the populations based on the morphometric and landmark distances data was drawn by the unweighted pair group (UPGMA) cluster analysis. Univariate analysis of variance (ANOVA) and independent sample $t$-test were carried out to test the significance of morphological differences. Comparison of meristic characters was done using non parametric Mann-Whitney U test. Resulting DFAs were examined for the extent of classification between two stocks and between stocks. In addition, a "leave-one-out cross-validation" was performed on each DFA as a testing procedure. All statistical analyses were done using SPSS v 11.5.

\section{Results}

Length and body weight of M. cuchia and $O$. bengalense and their sexing pattern

Their sex patterns were found to be heterosexual i.e. male and female in both the species. The sex and location wise length and body weight of $M$. cuchia and $O$. bengalense are presented in Table 1.

\section{Physical characteristics}

The body of $M$. cuchia is cylindrically elongated. The shape of body is triangular. The skin is very thick and rough looking. The dorsal part of body colour is dark brownish and ventral part is yellow brownish. All over the body is covered by numerous black blotches. The body of $O$. bengalense is rounded and elongated. Body shape is rounded. The dorsal part of body colour is lightly dark reddish brown and the ventral part is whitish red with very little minute like light blackish spot. The skin of body is so transparent and sees the light zigzag fashion line in the skin which began slightly far of chest (approximately $5 \mathrm{~cm}$ far) after death the line is more dominant.

The head shape of M. cuchia is triangular (Fig. 2a). Middle part of the head is slightly straight. Numerous lines and black blotches are present on the head. Eyes are small and visible through a translucent layer of skin. Mouth part is blunt shape. Upper jaw is so long due to large head size. The head is short and rounded in $O$. bengalense (Fig. 2b). Mouth part is sharp. Eyes are protractile and no line is present as like M. cuchia. The middle part of the head is anteriorly rounded. Upper jaw is not long due to short head size. At the upper part of jaw there have two small barbels.

At ventral part of head there are various line arranged ' $\mathrm{V}$ ' liked shape $M$. cuchia (Fig. 2c). More than 22-28 lines are arranged here. Lines are going to near the lower jaw. In the M. cuchia two sides of the head is looking large swollen like structure i.e. having respiratory sac. The ventral part of $O$. bengalense there have around 10 line arranged with crescent shape (Fig. 2d). Lines are finally going to near the lower jaw. The two sides of the head are not looking swollen.

There are four lines present beside the lateral line in $M$. cuchia (Fig. 2e). There is no line present beside lateral line in O. bengalense (Fig. 2f).

The last part of the tail of M. cuchia is blunt shape (Fig. $2 \mathrm{~g}$ ). The dorsal and anal fin are rudimentary i.e. they are not show dominant. The dorsal fin is originated slightly away at the dorsal part from the anus. The last part of the tail of $O$. bengalense is something sharp like structure. The dorsal and anal fins are more dominant than $M$. cuchia. The dorsal fin is slightly before position from the anus (Fig.2h). The gill arch is spiral-like and fiber-like fashion in M. cuchia (Fig. 2i) and O. bengalense (Fig. 2j), respectively. The mouth gape is broad in M. cuchia (Fig. $2 \mathrm{k}$ ) and in $O$. bengalense (Fig. 21), it is not so broad as $M$. cuchia. The teeth of $M$. cuchia (Fig. $2 \mathrm{~m}$ ) are relatively large and are countable compared to $O$. bengalense. The teeth of $O$. bengalense (Fig. 2n) are very small in size and are not countable. In the stomach of $M$. cuchia, no other structure is attached with intestine. In case of $O$. bengalense, fat like structure is attached with intestine (Fig. 2o).

\section{Meristic characters}

The mean number of line in body, number of line below head, number of teeth (upper jaw), number of teeth (lower jaw) and number of gill raker were $6.0 \pm 0$, $21.94 \pm 2.06, \quad 17.47 \pm 2.97, \quad 26.66 \pm 4.57$ and 8.0 \pm 0 , respectively in $M$. cuchia. Whereas the mean number of line below head and gill raker was 10.21 \pm 0.631 and $8.0 \pm 0$, respectively in $O$. bengalense. The mean number of line below head were significantly (Mann-Whitney U test; $\mathrm{z}=-6.091 ; P<0.001)$ different between two species. 


\section{Morphological and landmark differences}

Among the thirty four transformed morphometric (26 characters) and truss measurements (8 characters) of $M$. cuchia, two morphometric measurements (Eye diameter, $t$ $=-2.34 ; P<0.05$ and Pre orbital length, $t=-2.12 ; P<$ $0.05)$ and one truss measurement $(4-6, t=2.09 ; P<0.05)$ were found significantly different among the sexes of $M$. cuchia. Therefore, those characteristics were excluded for further analyses. Among the thirty two transformed morphometric (24 characters) and truss measurements $(8$ characters) of $O$. bengalense, two morphometric measurements (Lower jaw length, $t=2.73 ; P<0.05$ and Eye diameter, $t=-2.24 ; P<0.05$ ) were found significantly different among the sexes of $O$. bengalense. Therefore, those characteristics were excluded for discriminant analyses for both the species. None of the truss measurements were found significantly different between sexes of $O$. bengalense. One morphological character, fat length of $O$. bengalense was not found in $M$. cuchia and four lines present beside the lateral line in $M$. cuchia were not found in $O$. bengalense, therefore, those characters were not included for discriminannt analyses or to determine the difference between the species.

Independent sample $t$-test showed that fourteen morphometric characters, Pre dorsal length $(\mathrm{PDL}), t$-test $=$ 2.09; $P<0.05$; Pre anal length (PAL), $t-$ test $=3.35 ; P<$ 0.01 ; Post dorsal length (PoDL), $t$-test $=-5.86 ; P<0.001$; Post anal length( PoAL), $t$ - test $=-2.53 ; P<0.05$; Head length(HL), $t$-test $=2.39 ; P<0.05$; Snout length $(\mathrm{SnL}), t$ test $=2.71 ; P<0.01$; Upper jaw length $(\mathrm{UJL}) t$-test $=$ 4.15; $P<0.001$; Mouth gape(MG), $t$-test $=2.02 ; P<$ 0.05 ; Head width (HW) $t$ - test $=2.34 ; P<0.05$; Least body depth (LBD), $t$-test $=2.72 ; P<0.01$; Highest body diameter (HBD) $t$-test $=2.35 ; P<0.05$; Intestine length (IL) $t$ - test $=2.55 ; P<0.05$ and two truss measurement 3 $5, t$-test $=4.94 ; P<0.001 ; 5-6, t$-test $=-6.53 ; P<0.001$ revealed a significant variation between two species in varying degrees. Length of lateral line $(\mathrm{LAL}), t$-test $=$ $0.58 ; \mathrm{P}=0.568$; Head depth (HD), $t$-test $=-0.83 ; \mathrm{P}=$ 0.411; Post orbital length (PoOrL) $t$-test $=-1.19 ; \mathrm{P}=$ 0.242 ; Greatest body depth (GBD), $t$-test $=1.90 ; \mathrm{P}=$ 0.064; Greatest width of body depth ( GWD), $t$-test $=$ $0.74 ; \mathrm{P}=0.465$; Width of body at vent (WBV), $t$-test $=$ $0.93 ; \mathrm{P}=0.356$; Depth of body at vent $(\mathrm{DBV}), t$-test $=$ $1.31 ; \mathrm{P}=0.195 ;$ Distance between vent and commencement of dorsal fin $(\mathrm{DBCB}), t$-test $=-0.56 ; \mathrm{P}=$
0.578 and five truss measurement $1-2, t$-test $=-0.69 ; \mathrm{P}=$ $0.491 ; 1-3, t$-test $=-0.16 ; \mathrm{P}=0.878,2-3, t$-test $=0.15 ; \mathrm{P}=$ $0.884,2-4, t$-test $=0.03 ; \mathrm{P}=0.974,4-5, t$-test $=-0.93 ; \mathrm{P}=$ 0.355 did not varied significantly between two species.

Univariate statistics (ANOVA) showed that eleven morphometric characters revealed a significant variation between two species in varying degrees (Table 2). First three canonical discriminant functions were used in the analysis. Pooled within-groups correlations between discriminant variables and DFs revealed that four morphometric characters (PoDL, UJL, PAL and IL) and two landmark distances (5-6 and 3-5) contributed to the first DF and three morphometric characters (HBD, LBD and $\mathrm{MG}$ ) contributed second $\mathrm{DF}$ and six morphometric characters (HL, HBD, PoAL, SnL, PDL and LAL) contributed to the third implying that these characters are the most important in the description of population characteristics. Plotting Discriminant function DF1 and DF2 showed a clear differentiation between the species as well as between the stocks for both morphometric and landmark measurements. For both morphometric and landmark measurements the first and second DF accounted $64.8 \%$ and $33.2 \%$ of among group variability, explaining $98 \%$ of total group variability. All the populations were clearly separated from each other in the discriminant space (Fig. 3). This suggested that there was limited intermingling among populations and the populations of the species were separated.

A dendrogram based on morphometric and landmark distance data was shown for the populations of both the species constructed one cluster and further divided into two distinct sub-clusters. M. cuchia collected from Mymensingh and $M$. cuchia collected from Dinajpur constructed one sub-cluster and $O$. bengalense collected from Sathkhira and $O$. bengalense collected from Bagerhat constructed another sub-cluster based on the Distance of squared Euclidean dissimilarity (Fig. 4).

A correct classification of individuals into their original population from leave-one-out-classification varied between $93.3 \%$ and $94.1 \%$ by discriminant analysis and 95.9\% of individuals could be classified in their correct priori grouping. The proportion correctly classifies $M$. cuchia (Dinajpur) into the corresponding original group was the highest (93.3\%) (Table 3).

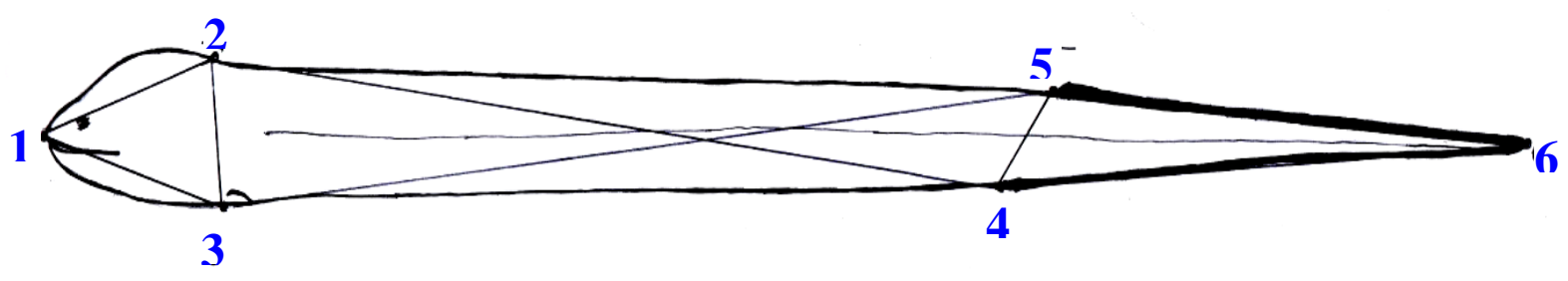

Fig.1. Six landmarks determining 8 distances on eel body 
Table 1. Length and body weight of $M$. cuchia and $O$. bengalense and their sexing pattern $(\mathrm{SD}=\mathrm{standard}$ deviation, $\mathrm{n}=$ number of fish)

\begin{tabular}{|c|c|c|c|c|c|}
\hline Species & Sex & $\begin{array}{c}\text { Location of } \\
\text { samples }\end{array}$ & $\mathrm{n}$ & $\begin{array}{l}\text { Mean total length } \pm \text { SD } \\
(\mathrm{cm})\end{array}$ & $\begin{array}{c}\text { Mean weight } \pm \text { SD } \\
(\mathrm{g})\end{array}$ \\
\hline \multirow[t]{4}{*}{ Monopterus cuchia } & $\mathrm{F}$ & 1 & 4 & $\begin{array}{c}70.15 \pm 4.45 \\
(66-74)\end{array}$ & $\begin{array}{c}825 \pm 28.87 \\
(800-850)\end{array}$ \\
\hline & M & & 13 & $\begin{array}{l}67.9 \pm 2.14 \\
(62-71)\end{array}$ & $\begin{array}{c}771.54 \pm 118.99 \\
(500-900)\end{array}$ \\
\hline & $\mathrm{F}$ & 2 & 9 & $\begin{array}{c}58.82 \pm 2.69 \\
(52-61)\end{array}$ & $\begin{array}{c}281.11 \pm 68.23 \\
(150-300)\end{array}$ \\
\hline & M & & 6 & $\begin{array}{l}55.5 \pm 4.07 \\
(52-63)\end{array}$ & $\begin{array}{l}274.17 \pm 74.39 \\
\quad(215-400)\end{array}$ \\
\hline \multirow[t]{4}{*}{ Ophisternon bengalense } & $\mathrm{F}$ & 3 & 6 & $\begin{array}{l}53.02 \pm 2.60 \\
(48-55)\end{array}$ & $\begin{array}{l}158.33 \pm 20.41 \\
(150-200)\end{array}$ \\
\hline & M & & 2 & $\begin{array}{c}100 \pm 0 \\
(48-48)\end{array}$ & $\begin{array}{c}47.6 \pm 0 \\
(100-100)\end{array}$ \\
\hline & $\mathrm{F}$ & 4 & 6 & $\begin{array}{l}53.72 \pm 5.96 \\
(46-63)\end{array}$ & $\begin{array}{l}175 \pm 27.39 \\
(150-200)\end{array}$ \\
\hline & M & & 3 & $\begin{array}{c}55.83 \pm 8.60 \\
(48-63)\end{array}$ & $\begin{array}{c}183.33 \pm 28.89 \\
(150-200)\end{array}$ \\
\hline
\end{tabular}

1. M. cuchia (Mymensingh), 2. M. cuchia (Dinajpur), 3. O. bengalense (Sathkhira), and 4. O. bengalense (Bagerhat)

\section{Discussion}

The present approached to quantify morphological characterization within eel population as well as between the species, with the goal of placing this characterization within a clear functional framework relating to landmark distance. Neotropical species of Synbranchidae are included in two genera: Synbranchus and Ophisternon. Among the Synbranchids, a few are known as a protogynous diandric fish (Nostro and Guerrero, 1996), with two different kinds of males. Collins et al. (2002) reported that swamp eel have high fecundity and are protogynous hermaphrodite. In this experiment according to sexing pattern, there were nineteen males (59.4\%), thirteen females $(40.6 \%)$ in $M$. cuchia and five males $(29.4 \%)$ and twelve females $(70.6 \%)$ in O. bengalense. There were minor sex-dependent morphological differences detected in the present study. Some groups, such as lampreys exhibit relatively little variation in body and head shape (Gatesy and Middleton, 1997), while other groups, such as teleost fishes and Hawaiian honeycreepers, display a spectacular variety of forms. In the present study, from the physical characteristics, the head shape of M. cuchia is broad and lip are blunt shape, but in $O$. bengalense the head is short and lip are sharp having two little barbels in upper lip. There are some line showed in head of $M$. cuchia and four lines are also present beside the lateral line. These lines are fully absent in $O$. bengalense. Eyes are protractile and visible from long distance in $O$. bengalense but in M. cuchia eye is small and visible only through a translucent layer of skin. Eyes are well developed, visible through a translucent layer of skin, and vertically aligned to posterior nostrils in Synbranchus lamperia (Favorito et al., 2005). The body length and weight are much greater in $M$. cuchia than in $O$. bengalense.

The average length and weight of $M$. cuchia were $62.74 \pm$ $6.84 \mathrm{~cm}$ and $547.03 \pm 271.48 \mathrm{~g}$. On the other hand the average length and weight of $O$. bengalense were $53.12 \pm$ $5.27 \mathrm{~cm}$ and $161.76 \pm 33.21 \mathrm{~g}$. There were differences in tail shape in the two species as well. In M. cuchia the last part of tail is blunt and cylindrical. The last part of the tail is sharp in $O$. bengalense. The dorsal and anal fin are rudimentary in $M$. cuchia but are well developed in $O$. bengalense. All over the body is triangular shape in M.cuchia and round in O.bengalense. Favorito et al. (2005) reported that $S$. lampreia can be easily distinguished from $S$. marmoratus and $S$. madeirae by its color pattern which consists of conspicuous large black or dark brown, roundish blotches scattered along body, rare on head, and a relatively lighter ground coloration with small and inconspicuous light brown spots vs. more homogeneously distributed spots and marbled pattern in $S$. marmoratus and gray uniform coloration with lighter roundish spots in $S$. madeirae. The phenotypic plasticity of fish is very high. They adapt quickly by modifying their physiology and behavior to environmental changes. These modifications ultimately change their morphology (Stearns, 1983). In the present study, both the eels look very similar to the common people and most of the times harvested and sold as same species but with a close observation, they look different phenotypically. The differences are well-exposed when the external and 
internal anatomy are studied. The body colour of $M$. cuchia brownish dark with numerous small black and
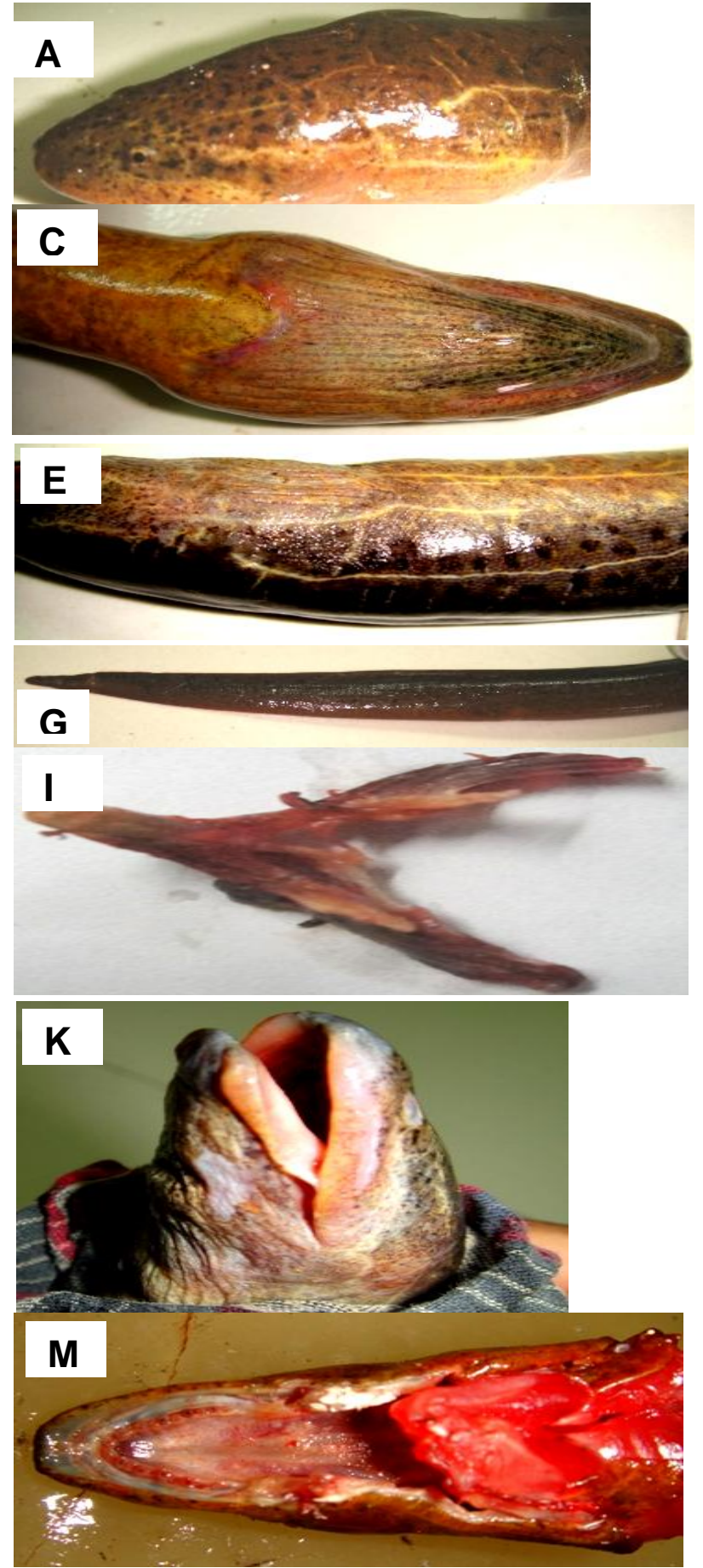

yellowish blotches and the color of head is blackish brown with numerous spots or line.
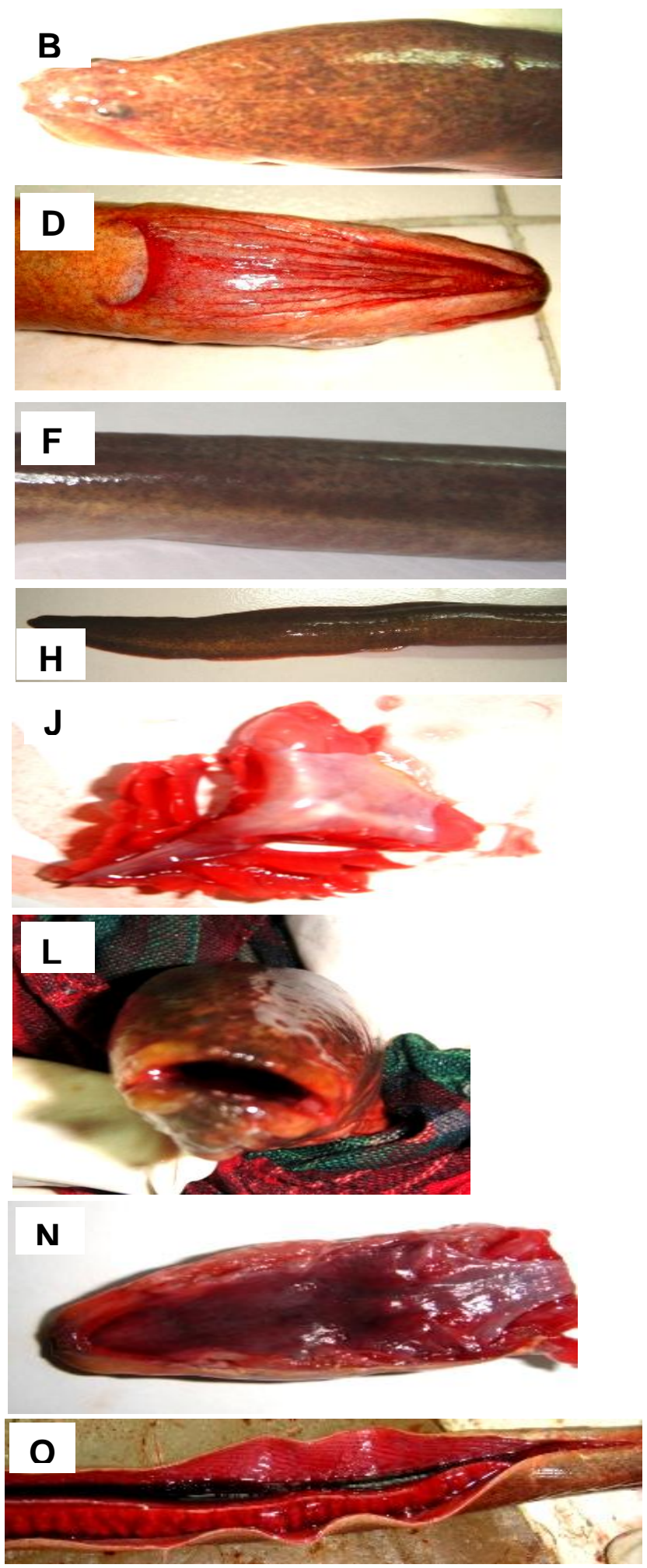

Fig. 2. Compare the morphology between two eels, M. cuchia (left) and O. bengalense (right) 


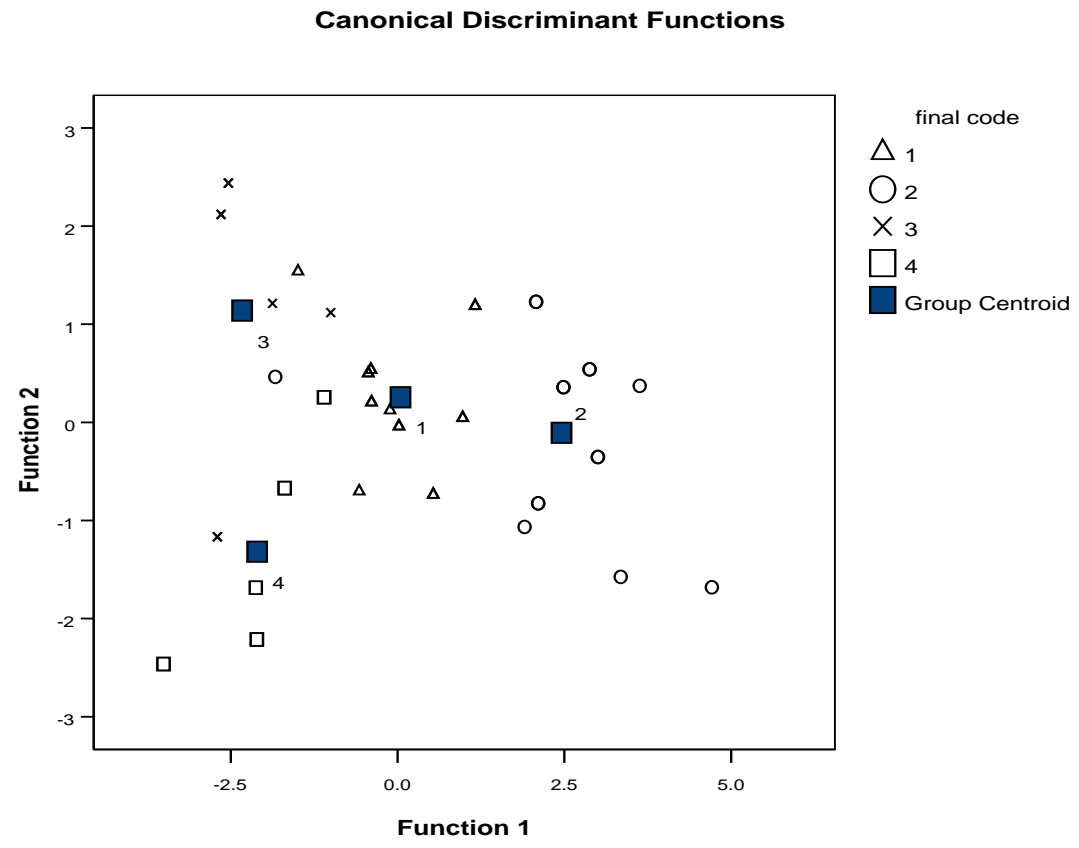

Fig. 3. Sample centroids of discriminant function scores based on morphometric and truss measurements, 1. M. cuchia (Mymensingh), 2. M. cuchia (Dinajpur), 3. O. bengalense ( Sathkhira), and 4. O. bengalense (Bagerhat)

Distance of squared Euclidean dissimilarity

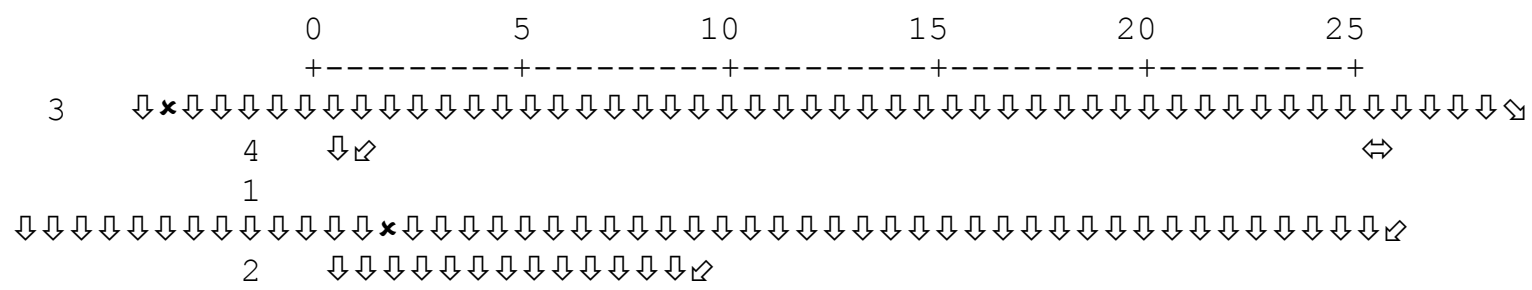

Fig. 4. Dendrogram based on morphometric characters and landmark distances

The body colour of M. cuchia collected from Dinajpur is slightly lighter than those collected from Mymensingh. The overall body colour of $O$. bengalense is brownish red, the ventral part is whitish red and the dorsal part is deep brown. Unlike $M$. cuchia in $O$. bengalense no black/yellowish small blotches are found. However, in $O$. bengalense the light-coloured zigzag line are found in both dorsal and ventral side of the body. The skin of $M$. cuchia is much thicker than the skin of $O$. bengalense. The present results demonstrated significant differences in morphometric characters among the populations of $M$. cuchia and $O$. bengalense. This morphometric differentiation also supported by the meristic traits. The number of scales, the number of gill rakers and both pectoral and pelvic-fin lengths as well as eye size and body height have been used as criteria for separating species (Norman, 1937). Meristic data did not support the homogeneity between $M$. cuchia and $O$. bengalense samples. The pattern of meristic data overlapping at number of gill raker. Nakamura (2003) found differences in meristic counts in Japanese charr (Salvelinus leucomaenis) among the river systems (Naka and Tone rivers, central Japan) and among the tributaries of the Naka River (Ashinagasawa, Akasawa, Ushirosawa and Moto-okashirasawa streams). However, in this experiment there are limited differences between two populations. For the meristic traits no. of line below head significantly differed in the populations. However, the other meristic traits are not significant among the populations. Meristic characters are limited significantly 
different from other characters because of species variation.

Table 2. Univariate statistics (ANOVA) testing difference between $M$. cuchia and $O$. bengalense $(\mathrm{df} 1=1 ; \mathrm{df} 2=47)$

\begin{tabular}{lccr}
\hline Characters & Wilks' Lambda & F & Significance \\
\hline PDL & 0.915 & 4.352 & $0.042^{*}$ \\
PoDL & 0.578 & 34.289 & $0 * * *$ \\
PAL & 0.807 & 11.216 & $0.002^{* *}$ \\
PoAL & 0.88 & 6.404 & $0.015^{*}$ \\
LAL & 0.993 & 0.331 & 0.568 \\
HL & 0.892 & 5.707 & $0.021^{*}$ \\
SnL & 0.865 & 7.326 & $0.009^{* *}$ \\
UJL & 0.732 & 17.219 & $0 * * *$ \\
MG & 0.92 & 4.089 & $0.049^{*}$ \\
HD & 0.986 & 0.687 & 0.411 \\
HW & 0.895 & 5.491 & $0.023^{*}$ \\
PoOrL & 0.971 & 1.405 & 0.242 \\
GBD & 0.929 & 3.603 & 0.064 \\
LBD & 0.864 & 7.406 & $0.009 * *$ \\
GWD & 0.989 & 0.543 & 0.465 \\
HBD & 0.895 & 5.539 & $0.023^{*}$ \\
WBV & 0.982 & 0.867 & 0.356 \\
DBV & 0.965 & 1.726 & 0.195 \\
DBCB & 0.993 & 0.314 & 0.578 \\
IL & 0.879 & 6.485 & $0.014 *$ \\
1-2 & 0.99 & 0.481 & 0.491 \\
1-3 & 0.999 & 0.024 & 0.878 \\
2-3 & 1 & 0.021 & 0.884 \\
2-4 & 1 & 0.001 & 0.974 \\
$3-5$ & 0.658 & 24.392 & $0 * * *$ \\
$4-5$ & 0.982 & 0.871 & 0.355 \\
$5-6$ & 0.525 & 42.58 & $0 * * *$ \\
\hline$*<0.05 ; * * P 0.01 ; * * * P<0.001$ & &
\end{tabular}

Table 3. Correct classifications of individuals M. cuchia (collected from Mymensingh and Dinajpur) and O. bengalense (collected from Sathkhira and Bagerhat) into their original population (leave-one-out-classification)

\begin{tabular}{|c|c|c|c|c|c|c|}
\hline & \multirow{2}{*}{ Population } & \multicolumn{4}{|c|}{ Predicted Group Membership } & \multirow[t]{2}{*}{ Total } \\
\hline & & 1 & 2 & 3 & 4 & \\
\hline \multirow[t]{4}{*}{ Original Count } & 1 & 16 & 0 & 1 & 0 & 17 \\
\hline & 2 & 1 & 14 & 0 & 0 & 15 \\
\hline & 3 & 0 & 0 & 8 & 0 & 8 \\
\hline & 4 & 0 & 0 & 0 & 9 & 9 \\
\hline \multirow[t]{4}{*}{$\%$} & 1 & 94.1 & 0 & 5.9 & 0 & 100 \\
\hline & 2 & 6.7 & 93.3 & 0 & 0 & 100 \\
\hline & 3 & 0 & 0 & 100 & 0 & 100 \\
\hline & 4 & 0 & 0 & 0 & 100 & 100 \\
\hline
\end{tabular}

1. M. cuchia (Mymensingh), 2. M. cuchia (Dinajpur), 3. O. bengalense (Sathkhira), and 4. O. bengalense (Bagerhat)

As morphology is especially dependent on environmental conditions during early life-history stages (Ryman et al., 1984; Lindsey, 1988; Cheverud, 1988), phenotypic differentiation may indicate that the majority of fish spend their entire lives in separate regions. Morphometric differences among stocks are expected, because they are geographically separated and may have originated from different ancestors. Therefore, it is not unlikely that obvious environmental variations exist in eels collected from four locations (Mymensingh, Dinajpur, Satkhira and Bagerhat). Fish are very sensitive to environmental changes and quickly adapt themselves by changing necessary morphometrics. It is well-known that morphological characters can show high plasticity in 
response to differences in environmental conditions, such as food abundance and temperature (Allendorf and Phelps, 1988; Swain et al., 1991; Wimberger, 1992). In the study, fish demonstrate greater variances in morphological traits both within and between populations and are susceptible to environmentally induced morphological variations. Morphometric measurements were submitted as the percentage of the total length (TL) and the differences among the populations were tested by ANOVA. Morphometric measurements and meristic counts were subjected to stepwise discriminant function analysis (DFA). Among the thirty four transformed morphometric (26 characters) and two morphometric measurements, pre anal length and width of body at vent and one truss measurement (4-6) were found significantly different among the sexes of $M$. cuchia. Other characters did not show significant differences. The results are similar to those reported by Cakmak and Ahmet (2010) for freshwater eel M. mastacembelus. Among the thirty two transformed morphometric (24 characters) and truss measurements (8 characters), six morphometric measurements - mouth gap, eye diameter, head depth, head width, greatest width of body depth, intestine length were found significantly different among the sexes of $O$. bengalense. None of the truss measurements were found significantly different between sexes of $O$. bengalense. Therefore, stock identification based on morphological characters must be confirmed by genetic evidence to verify that the phenotypic differences reflect some degree of reproductive isolation rather than simply environmental differences. On the other hand, stock discrimination by morphologic markers might be appropriate for fisheries management even if this phenotypic divergence is not reflected by genetic differentiation.

To our knowledge, there are no reports on the morphological characterization between two eels $M$. cuchia and $O$. bengalense till now. To elucidate the facts, the discriminant analysis on morphological variation between two populations of cuchia was conducted along with ANOVA with every morphological character parameters of them. Univariate statistics (ANOVA) showed that eleven morphometric characters and one truss measurement (3-5) revealed a significant variation between two species in varying degrees. Five morphometric characters and four truss measurements did not varied significantly between two species. Morphometric and meristic analyses showed a similar pattern of differentiation between Scomber japonicus stocks and revealed a clear discreteness of two groups, northeastern Mediterranean (Antalya Bay-Iskenderun Bay) and the northern group, including the Aegean, Marmara, and Black Seas (Erguden et al., 2009).

Truss network systems are a powerful tool for identifying stocks of fish species (Turan et al., 2004). An unbiased network of morphometric measurements over a 2 dimensional outline of a fish removes the need to find the types of characters and optimal number of characters for stock separation, and provides information over the entire fish form (Turan et al., 2004). The truss network system can effectively be used to distinguish between the stocks. In the present study one truss measurement (4-6) was found significantly different among the sexes of $M$. cuchia. In this study, morphometric character and truss parameters were combined and used to diffrentiate the populations of two eels. For both morphometric and landmark measurements the first and second DF accounted $82.8 \%$ and $13.0 \%$ of among group variability, explaining $94.1 \%$ of total group variability. It proved that discriminant analysis as an effective method in identifying populations, strains, and subspecies which have near relations.

Plotting DFs revealed high isolation in morphometrics among the stocks. Discrimination of the three morphotypes was confirmed statistically by the significant difference between population centroids and by the percentage of reallocation. The results of the study are useful as baseline information of $M$. cuchia and $O$. bengalense populations for further studies. As the result of this study shows all the studied populations were separated from each other in the discriminant space. This suggested that there was limited intermingling among populations and the populations of the species were separated.

The dendrogram employed in this study resulted in the populations of both the species constructed one cluster and further divided into two distinct sub-clusters. $M$. cuchia collected from Mymensingh and from Dinajpur constructed one sub-cluster and $O$. bengalense collected from Sathkhira and from Bagerhat constructed another sub-cluster based on the Distance of squared Euclidean dissimilarity. The difference between the stocks may have been due to environmental as well as genetic variations. A correct classification revealed variation into their original population between $93.3 \%$ and $94.1 \%$. Turan et al. (2006) observed that the pooling of the winter and summer samples of same species of the same areas in reclassification analysis also supported the same pattern of differentiation among samples.

Finally it is obvious that due to the observed high phenotypic discreteness in relation to geography, the stocks of $M$. cuchia and $O$. bengalense are separated from one another.

\section{Conclusions}

It is widely recognized that morphometric characters are diagnostically useful when the number of specimens examined is adequate and the methods can be statistically tested. Therefore, morphological characterization is very useful tool for differentiation of stocks, to know population structure and finally for further genetic 
analysis. Both eels have high commercial value with domestic and overseas demand and their biodiversity should be conserved and should be brought under aquaculture to save them from extinction.

\section{References}

ADCP. 1995. Development of coastal aquaculture in the Mediterranean region. Rome, FAO, ADCP/MR, 6.

Allendorf, F. W. and Phelps, S. R. 1988. Loss of genetic variation in hatchery stock of cutthroat trout. Trans. Am. Fish. Soc., 109:537-543.

August, S. M. and Hicks, B. J. 2006. Glass eel recruitment and environmental conditions in the Tukituki River, New Zealand. Environ. Biol. of Fishes, 12(2):117-118.

BOBP. 1985. Marine Small-scale Fisheries of Bangladesh: A General Description. FAO/Bay of Bengal, Madras, India.

Breder, C. M. and D. E. Rosen, 1966. Modes of reproduction in fishes. T. F. H. Publications, Neptune City, New Jersey. 941 p.

Cakmak, E. and Ahmet, A. 2010. Morphological differences among the Mesopotamian spiny eel, Mastacembelus mastacembelus (Banks \& Solander 1794), Popul. Turk. J. Fish. Aquat. Sci., 10:87-92.

Cheverud, J. M. 1988. A comparision of genetic and phenotypic correlations. Evolution. 42:958-968.

Chowdhury, W. N.; Khan, M. G.; Myklevoll, S. and Saetre, R. 1980. Preliminary results from a survey on the marine fish resources of Bangladesh November December 1979. Institute of Marine Research, Bergen.

Collins, T. M.; Trexler, J. C.; Nico, L. G. and Rawlings T. A. 2002. Genetic diversity in a morphologically conservative invasive taxon: multiple introductions of swamp eels to the Southeastern United States. Conserv. Biol., 16(4):1024-1035.

Elliott, N. G.; Haskard, K. and Koslow, J. A. 1995. Morphometric analysis of orange roughy (Hoplostethus atlanticus) off the continental slope of southern Australia. J. Fish. Biol., 46:202-220.

Erguden, D.; Bayram, O.; Erdogan, Z. A. and Turan, C. 2009. Morphologic structuring between populations of chub mackerel Scomber japonicus in the Black, Marmara, Aegean and northeastern Mediterranean Seas. Fish Sci., 75: 129-135.

FAO. 2005. Fishery statistics; Aquaculture production (2004). FAO Fisheries series No. 72/FAO Statistics No.185, Rome, Italy.

FAO/UN. 2005. The markets for the prime Mediterranean species sea bass, sea bream, mullets and eel and their links with investment. ADCP/REP, 28:26.

Favorito, S. E.; Zanata, A. M. and Assumpcao, M. I. 2005. A new Synbranchus (Teleostei: Synbranchiformes: Synbranchidae) from ilha de Marajó, Pará, Brazil, with notes on its reproductive biology and larval development. Neotrop. ichthyol., 3(3):319-328.

FRSS. 1984. Fisheries Resources Survey System Project. Department of Fisheries, Government of Bangladesh.

Gatesy, S. M. and Middleton, K. M. 1997. Bipedalism, flight, and the evolution of theropod locomotor diversity. J. Vertebr. Paleontol.,17(2):308-329.

Hicks, B. J. and McCaughan, H. M. C. 1997. Land use, associated eel production, and abundance of fish and crayfish in streams in Waikato, New Zealand. New Zealand J. Marine and Freshw. Res., 31:635-650.

Hossain, M. A. R.; Nahiduzzaman, M.; Saha, D.; Khanam, M. U. H. and Alam, M. S. 2010. Landmark-based morphometric and meristic variations of the endangered carp, Kalibaus Labeo calbasu, from stocks of two isolated rivers, the jamuna and halda and a hatchery. Zool. Stud., 49: 556-563.

Ishak, 1994. Market power, vertical linkages and government policy: the fish industry in Peninsular Malaysia. Oxford University Press. UK.

Jessop, B. M. 2000. The American eel. Fisheries and Oceans Canada, Ottawa, Ontario, 8p.

Jhingran, A. G. and Talwar, P. K. 1991. Inland Fisheries of India and adjacent countries. Oxford and IBH publishing co. pvt. Ltd. Calcutta. 514p.

Lindsey, C. C. 1988. Factors controlling Meristic variation. In: Hoar, W. S. and Randall, D. J. (eds.), Fish Physiology, 11-B. Academic Press, San Diego, CA, 197-274.

Mazid, M. A. 2002. Development of Fisheries in Bangladesh: Plans and Strategies for Income Generation and Poverty Alleviation, 43-46.

Moriarty, C. and Dekker, W. 1997. Management of the European eel. 1st ed. Fish. Bull., (Dublin) 15:110.

Nakamura, T. 2003. Meristic and morphometric variations in fluvial Japanese char between river systems and among tributaries of a river system. Environ. Biol. of Fishes, 66:133-141.

Norman, J. R. 1937. Coast fishes. Part II. The Patagonian region. Discovery Reports, (Cambridge) 16:1-76.

Rahman, A. K. A. 2005. Freshwater fishes of Bangladesh, 2nd ed. Dhaka, Bangladesh: Zoological Society of Bangladesh.

Ryman, N.; Lagercrantz, U.; Andersson, L.; Chakraborty, R. and Rosenberg, R. 1984. Lack of correspondence between genetic and morphologic variability patterns in Atlantic herring (Clupea harengus). Heredity, 53: 687-704.

Stearns, S. C. 1983. A natural experiment in life-history evolution: field data on the introduction of mosquitofish (Gambusia affinis) to Hawaii. Evolution, 37:601-617.

Swain, D. P.; Ridell, B. E. and Murray, C. B. 1991. Morphological Differences between Hatchery and Wild Populations of Coho Salmon (Oncorhynchus 
kisutch): Environmental Versus Genetic Origin. Can. J. Fish. Aquat. Sci., 48:1783-1791.

Turan, C.; Erguden, D.; Gurlek, M.; Basusta, N. and F Turan. 2004. Morphometric structuring of the anchovy (Engraulis encrasicolus L.) in the Black, Aegean and northeastern Mediterranean Seas. Turk. J. Vet. Anim. Sci., 28:865-871.

Turan, C.; Mustafa, O.; Bayram, O. and Duzgunes, E. 2006. Morphometric and meristic variation between stocks of Bluefish (Pomatomus saltatrix) in the
Black, Marmara, Aegean and northeastern Mediterranean Seas. Fish. Res., 79(1-2): 139-147.

Usui, A. 1991. Eel Culture, 2nd ed. Fishing News Books. Blackwell Scientific Publications Inc, Oxford.

VAC (Victorian Aquaculture Council). 1999. Eels Aquaculture. Industry Sector Profile, Melbourne.

Wimberger, P. H. 1992. Plasticity of fish body shape - the effects of diet, development, family and age in two species of Geophagus (Pisces: Cichlidae). Biol. J. Linn. Soc., 45:197-218. 